\title{
Pre-ischemic exercise alleviates oxidative damage following ischemic stroke in rats
}

\author{
RUI FENG ${ }^{1 *}$, MIN ZHANG $^{2 *}$, XIAO WANG ${ }^{1 *}$, WEN-BIN LI ${ }^{2}$, SHI-QING REN ${ }^{1}$ and FENG ZHANG ${ }^{3,4}$ \\ ${ }^{1}$ Department of Neurology, The Third Hospital of Hebei Medical University, Shijiazhuang, Hebei 050051; \\ ${ }^{2}$ Department of Pathophysiology, Hebei Medical University, Shijiazhuang, Hebei 050017; \\ ${ }^{3}$ Department of Rehabilitation Medicine; ${ }^{4}$ Hebei Provincial Orthopedic Biomechanics Key Laboratory, \\ The Third Hospital of Hebei Medical University, Shijiazhuang, Hebei 050051, P.R. China
}

Received January 17, 2014; Accepted July 8, 2014

DOI: $10.3892 / \mathrm{etm} .2014 .1874$

\begin{abstract}
Physical exercise has been proved to be neuroprotective in clinical trials and animal experiments. However, the exact mechanism underlying this neuroprotective effect remains unclear. The aim of the present study was to explore whether pre-ischemic treadmill training could act as a form of ischemic preconditioning in a rat following ischemic stroke by reducing oxidative damage. Fifty-four rats were randomly divided into three groups ( $\mathrm{n}=18$ per group): Sham surgery, middle cerebral artery occlusion (MCAO) without exercise and MCAO with exercise. Subsequent to treadmill training, ischemic stroke was induced by occluding the MCA for $1.5 \mathrm{~h}$, followed by reperfusion. Six rats in each group were evaluated for neurological deficits and then sacrificed by decapitation to calculate the infarct volume. The remaining rats in each group were sacrificed to detect the level of superoxide dismutase (SOD) activity $(n=6)$ and malondialdehyde (MDA) concentration $(n=6)$. The results indicated that pre-ischemic exercise training reduced brain infarct volume and neurological deficits, increased SOD activity and decreased the concentration of MDA following ischemic stroke. In conclusion, treadmill exercise training prior to $\mathrm{MCAO} /$ reperfusion increased the antioxidant ability and decreased the oxidative damage in the brain subsequent to ischemic stroke.
\end{abstract}

Correspondence to: Dr Feng Zhang, Department of Rehabilitation Medicine, The Third Hospital of Hebei Medical University, 139 Ziqiang Road, Shijiazhuang, Hebei 050051, P.R. China E-mail: zjk20019@126.com

*Contributed equally

Key words: pre-ischemic exercise, oxidative damage, ischemic stroke, malondialdehyde, superoxide dismutase

\section{Introduction}

In the past two decades, animal experiments have indicated the protective effects of physical exercise on ischemic stroke, including enhanced survival rates, a reduction in neurological deficits, an alleviation of blood-brain barrier (BBB) dysfunction and an improvement in neurovascular integrity (1-5). Therefore, exercise preconditioning has begun to attract increasing attention.

Our previous review summarized the association between exercise preconditioning and brain ischemic tolerance, involving a series of pathological changes following ischemic stroke (6). However according to the search results at that time, there was no study on the antioxidative effect of pre-ischemic exercise following cerebral ischemia.

Regular exercise training has been shown to downregulate levels of free radicals (7), thus reducing the peroxidation levels of lipids or proteins in the brain of the rat (8). Furthermore, regular exercise training promoted the effect of antioxidant enzymes, including superoxide dismutase (SOD) and glutathione peroxidase (9-11). Since malondialdehyde (MDA) plays a key role in the process of ischemic stroke, the concentration of MDA in brain tissues and plasma has been used to assess the severity of neuronal ischemic injury (12-14).

A recent study indicated that pre-ischemic treadmill training for three weeks could alleviate brain oxidative damage by suppressing 4-hydroxy-2-nonenal-modified proteins and 8-hydroxy-2'-deoxyguanosine following ischemic stroke (15). In the study, the SOD activity in the exercise plus sham group was significantly higher than that in the sham group. However, whether pre-ischemic exercise can regulate the ischemic stroke-induced changes in SOD activity and MDA level remains unknown. Thus, the aim of the present study was to explore whether exercise preconditioning can regulate SOD and MDA and thereby decrease oxidative stress following MCAO.

\section{Materials and methods}

Animals. Fifty-four male Sprague Dawley rats, each weighing 200-220 g, were obtained from the Hebei Province Laboratory 
Animal Center (Shijiazhuang, China). All the rats were kept under a 12-h light/dark cycle. Food and water were available ad libitum. All the procedures in this study were approved by the Animal Care and Use Committee of Hebei Medical University (Shijiazhuang, China).

Treadmill training. Rats were randomly divided into three groups ( $\mathrm{n}=18$ per group): Sham surgery, middle cerebral artery occlusion (MCAO) without exercise and MCAO with exercise. Prior to formal training, rats in the MCAO with exercise group underwent adaptive running exercise training for two days at a speed of $5-8 \mathrm{~m} / \mathrm{min}$ for $30 \mathrm{~min} /$ day on a treadmill training machine (DSPT-202 Type 5-Lane Treadmill; Litai Biotechnology Co., Ltd., Shishi, China). Following the adaptive exercise training, the rats underwent formal treadmill training at a speed of $20 \mathrm{~m} / \mathrm{min}, 30 \mathrm{~min} /$ day for six days every week. The rats in the sham and MCAO without exercise groups did not receive exercise but were allowed to run freely in their living cages during the same time period.

MCAO model. Following the treadmill training, rats received MCAO surgery. Animals were anesthetized using $4 \%$ chloral hydrate $(10 \mathrm{ml} / \mathrm{kg}$, intraperitoneal) and were administered further doses if necessary to maintain the anesthesia state during the process of surgery. The body temperature of the rat was maintained at $37^{\circ} \mathrm{C}$ by a heating pad. The surgical procedures were performed in accordance with those described by Longa et al (16) with minor modification.

Briefly, the left external carotid artery (ECA), common carotid artery (CCA) and internal carotid artery (ICA) were firstly exposed. A monofilament (4-0 nylon suture) with a blunted poly-L-lysine coated tip (Beijing Sunbio Biotech Co., Ltd., Beijing, China.) was lightly inserted into the ECA. The suture then moved through the CCA and ICA, and finally occluded the MCA at its origin. After 90 min of MCAO, reperfusion was performed by removing the filament.

For the sham group, the CCA, ECA and ICA underwent the same procedures without occlusion of the MCA. The associated physiological parameters were monitored by a Blood Gas and Electrolyte System (ABL505; Radiometer Medical ApS, Copenhagen, Denmark). Rats were assessed $24 \mathrm{~h}$ after reperfusion according to a widely accepted scale as follows: 0 , no neurological symptoms; 1 , unable to completely extend the front jaw on the contralateral side; 2 , rotating while crawling and falling to the contralateral side; 3 , unable to walk without assistance; and 4, unconsciousness (16).

Determination of brain infarct volume. Twenty-four hours after reperfusion, animals were sacrificed by decapitation under chloral hydrate (10\%) anesthesia. The whole brains were stored in a refrigerator at $-20^{\circ} \mathrm{C}$ for $10 \mathrm{~min}$, following which each brain was cut into six coronal sections $(2 \mathrm{~mm}$ thick) between the anterior pole and the optic chiasm in the center. All tissues were immediately infiltrated into a $2 \%$ 2,3,5-triphenyltetrazolium chloride solution at $37^{\circ} \mathrm{C}$ thermostat for $30 \mathrm{~min}$, then fixed in $4 \%$ paraformaldehyde buffer. After $24 \mathrm{~h}$, a digital camera (DC240; Kodak, Rochester, NY, USA) and imaging software (Adobe Photoshop 7.0; Adobe Systems Inc., San Jose, CA, USA) were used to capture images and calculate the infarc- tion area. The total infarction volume was equal to the sum of the infarct area in each section. In order to minimize the error caused by brain edema, the corrected formula to calculate infarct volume was as follows: Infarct volume $=$ contralateral hemisphere region - non-infarcted region in the ipsilateral hemisphere. The following formula was used to calculate infarct percentage: Infarct percentage $=$ infarct volume/volume of the contralateral hemisphere $\mathrm{x} 100 \%$

Preparation of brain tissue. Twenty-four hours after reperfusion, the left hemisphere of the rat brain was harvested following perfusion transcardially with $0.9 \%$ saline under deep anesthesia. Briefly, the tissues were immersed into radio-immunoprecipitation assay lysis buffer and homogenized mechanically at $37^{\circ} \mathrm{C}$. The homogenate was centrifuged at $10,000 \mathrm{x} \mathrm{g}$ for $4 \mathrm{~min}$ at $4^{\circ} \mathrm{C}$ and the supernatant was collected. Protein concentration was measured using an Enhanced Bicinchoninic Acid Protein Assay kit (Beyotime Institute of Biotechnology, Haimen, China).

SOD activity detection. SOD activity in the brain tissue was detected using the methodology described by Oyanagui (17) according to the kit instructions (Nanjing Jiancheng Bioengineering Institute, Nanjing, China). The main principle was as follows: Superoxide anions were generated in the xanthine and xanthine oxidase system. These superoxide anions oxidized hydroxylamine, resulting in the formation of nitrite. This nitrite reacted with sulfanilic acid and naphthalene diamine, generating a colored product (18). SOD in the brain tissue decreased the superoxide anion concentration, thus reducing the colorimetric signal and absorbance, which was generally measured at $550 \mathrm{~nm}$. One unit of SOD activity was defined as the amount of enzyme that was required to inhibit the $50 \%$ reduction of nitroblue tetrazolium in the specified conditions. The procedures were repeated three times in order to obtain the mean values.

MDA assay detection. The concentration of MDA was detected by a thiobarbituric acid reaction method (19). Thiobarbituric acid reacts with MDA in acidic medium resulting in a pink-colored pigment at $95^{\circ} \mathrm{C}$. The absorbance at a wavelength of $532 \mathrm{~nm}$ was detected by a microplate reader (DU640; Beckman Coulter Inc., Miami, FL, USA). The procedures were performed based on the kit instructions (Nanjing Jiancheng Bioengineering Institute). MDA content ( $\mathrm{nmol} / \mathrm{mg}$ protein) was calculated using the following formula: Absorbance of sample tube/absorbance of standard tube $\mathrm{x} 2.5$. The procedures were repeated three times in order to obtain the mean values.

Statistical analysis. Statistical analysis was performed by SPSS for Windows, version 15.0 (SPSS Inc., Chicago, IL, USA). The neurological deficit scores and infarct volume between ischemic rats with and without pre-ischemic exercise were compared by an independent Student's t-test. The differences in SOD enzyme activity units and MDA concentration among the three groups were analyzed by one-way analysis of variance followed by a post hoc least significant difference test. Data are presented as the mean \pm standard deviation. $\mathrm{P}<0.05$ was considered to indicate a statistically significant difference in all statistical assessments. 


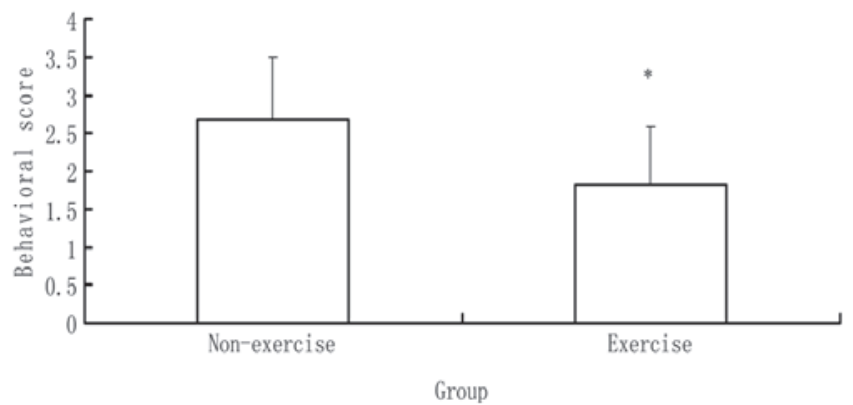

Figure 1. Behavioral scores of the MCAO with and without exercise groups. Data are presented as the mean \pm standard deviation $(n=6)$. The behavioral scores for the sham group were all zero (data not shown). "P<0.05 vs. the $\mathrm{MCAO}$ without exercise group. MCAO, middle cerebral artery occlusion.

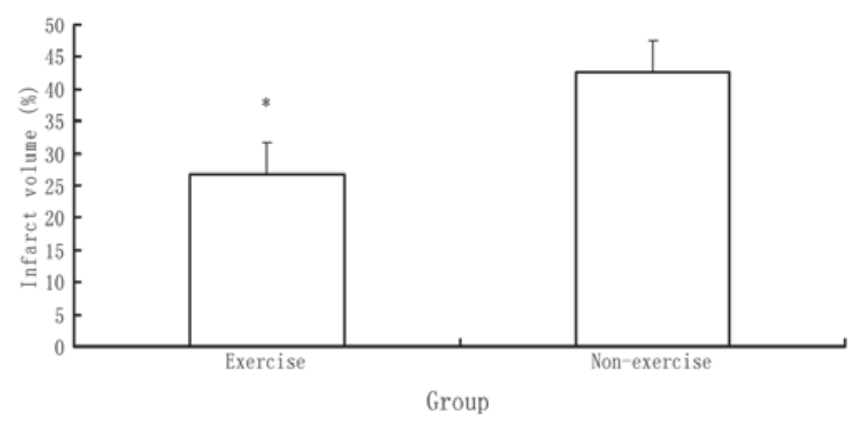

Figure 2. Difference in infarct volume between the MCAO with and without exercise groups. Data are presented as the mean \pm standard deviation. The infarct volume of the sham surgery group of six rats was zero (data not shown). ${ }^{\mathrm{P}}<0.05$ vs. the MCAO without exercise group. MCAO, middle cerebral artery occlusion.

\section{Results}

Physiological variables. Fifty-four rats were divided into three groups ( $n=18$ per group): Sham surgery, MCAO without exercise and MCAO with exercise. No significant differences were observed in the partial pressure of oxygen in arterial blood, the partial pressure of carbon dioxide in arterial blood or $\mathrm{pH}$ (hydrogen ion concentration) values among the three groups ( $\mathrm{P}>0.05$; data not shown).

Behavioral scores. Rats in the three groups were evaluated $24 \mathrm{~h}$ after reperfusion. In the sham surgery group, the rats exhibited no neurological symptoms. By contrast, a significant difference in behavioral scores was identified between the MCAO with and without exercise groups $(\mathrm{P}<0.05)$, as shown in Fig. 1. The rats in the MCAO with exercise group showed fewer neurological deficits than those in the MCAO without exercise group.

Infarct volume. Subsequent to behavioral evaluation, six rats in each group were sacrificed by decapitation to determine the infarct volume. The rats in the sham surgery group showed no ischemic areas. By contrast, a significant difference in infarct volume was identified between the MCAO with and without exercise groups $(\mathrm{P}<0.05)$, as indicated in Fig. 2. The rats in the MCAO with exercise group showed a significantly reduced ischemic area in the brain relative to those in the MCAO without exercise group.

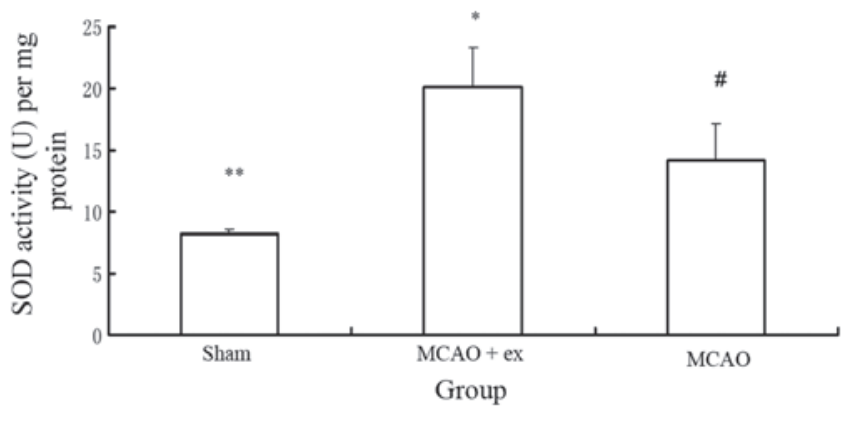

Figure 3. Difference in SOD activity among the three groups. The SOD activity of the MCAO with exercise group was higher than that of the MCAO without exercise group. The MCAO with and without exercise groups showed a higher SOD activity than the sham surgery group. Data are presented as the mean \pm standard deviation. ${ }^{*} \mathrm{P}<0.05$ vs. the sham surgery group; ${ }^{* *} \mathrm{P}<0.05$ vs. the MCAO with exercise group; ${ }^{*} \mathrm{P}<0.05$ vs. the MCAO without exercise group. MCAO, middle cerebral artery occlusion; MCAO + ex, MCAO with exercise; SOD, superoxide dismutase.

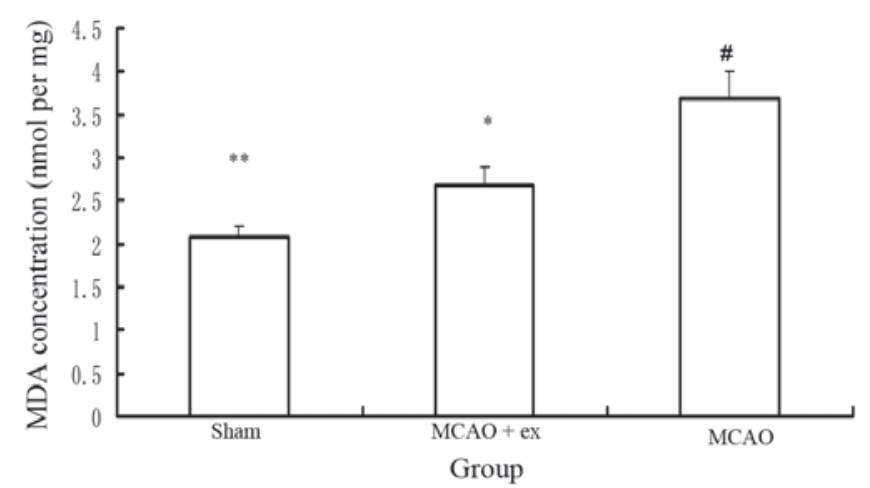

Figure 4. Difference in MDA concentration among the three groups. The MDA concentration of the MCAO with exercise group was lower than that of the MCAO without exercise group. The MCAO with and without exercise groups showed a higher MDA concentration than the sham surgery group. Data are presented as the mean \pm standard deviation. ${ }^{\#} \mathrm{P}<0.05$ vs. the sham surgery group; ${ }^{* *} \mathrm{P}<0.05$ vs. the $\mathrm{MCAO}$ with exercise group; ${ }^{*} \mathrm{P}<0.05$ vs. the MCAO without exercise group. MCAO, middle cerebral artery occlusion; MCAO + ex, MCAO with exercise; MDA, malondialdehyde.

SOD activity. Six rats in each group were sacrificed by decapitation to determine the SOD activity. A significant difference in SOD activity was identified among the three groups $(\mathrm{P}<0.05)$, as indicated in Fig. 3. The rats in the MCAO with exercise group showed a higher SOD activity in the brain relative to those in the MCAO without exercise group $(\mathrm{P}<0.05)$. The rats in the MCAO with and without exercise groups showed higher SOD activity in the brain relative to those in the sham surgery group $(\mathrm{P}<0.05)$.

MDA concentration. Six rats in each group were sacrificed by decapitation to determine the MDA concentration. A significant difference in MDA concentration was identified among the three groups $(\mathrm{P}<0.05)$, as indicated in Fig. 4. The rats in the MCAO with exercise group exhibited a lower MDA concentration in the brain relative to those in the MCAO without exercise group $(\mathrm{P}<0.05)$. The rats in the MCAO with and without exercise groups showed a higher MDA concentration in the brain relative to those in the sham surgery group $(\mathrm{P}<0.05)$. 


\section{Discussion}

Ischemic stroke is the third leading cause of mortality in Western countries (20). Therefore, strategies to prevent stroke and reduce brain damage following stroke have attracted increasing attention. A number of interventions have been investigated to achieve this, including physical activity (21).

A series of clinical studies on human subjects have explored the association between pre-ischemic exercise and the risk of ischemic stroke. These studies have produced different results. Certain studies have demonstrated that pre-ischemic exercise results in improved functional outcomes following stroke and a reduction in the ischemic stroke risk (21-24). The mechanisms underlying the neuroprotective effects of exercise pretreatment on post-stroke function and the occurrence of stroke are not clear according to these epidemiological studies, but have been revealed to be associated with the effects of pre-ischemic exercise on metabolic pathways, blood pressure, blood cholesterol and glucose level (22-25). By contrast, a prospective clinical study has indicated that pre-ischemic exercise may reduce the occurrence of ischemic stroke, but does not alleviate dysfunction following ischemic stroke (25). Thus, on the basis of the above-mentioned clinical studies, it is perhaps difficult to conclude that pre-ischemic exercise provides beneficial effects on the pathogenesis of stroke; however, it appears that the majority of the associated evidence demonstrates that pre-ischemic exercise exerts a beneficial effect on both the occurrence of and functional outcomes following ischemic stroke.

With regard to the mechanism underlying the effect of preconditioning exercise following ischemic stroke, animal experiments reported that pre-ischemic exercise improved BBB function and enhanced basal lamina integrity subsequent to ischemic stroke (26). Additionally, pre-ischemic exercise for three weeks increased cerebrovascular integrity in the striatum of rats $(27,28)$. Our previous studies demonstrated that exercise preconditioning reduced the over-release of glutamate and influenced glutamate receptor changes, alleviating brain damage subsequent to stroke $(29,30)$.

All the above-mentioned neuroprotective effects of pre-ischemic exercise may be indirectly associated with the antioxidant ability of exercise pretreatment following ischemic stroke. It is logically speculated that pre-ischemic exercise may alleviate BBB dysfunction, reduce glutamate over-release and increase cerebrovascular integrity so as to reduce oxidative stress following cerebral ischemia.

Lipid peroxidation is induced by high levels of free radicals, which can be caused by the cerebral ischemia-reperfusion (31-33). Long-term exercise training increases the antioxidant abilities of brain tissue (34). The present data also demonstrated that pre-ischemic exercise increased SOD activity and decreased MDA levels, thus providing a protective effect on oxidative injury following MCAO. The pre-ischemic exercise also decreased infarct volume and neurological deficits. However, further study is required to explore the mechanism underlying the antioxidant effect of pre-ischemic exercise training following ischemic stroke.

In conclusion, the results in the present study indicated that treadmill training exercise prior to $\mathrm{MCAO} /$ reperfusion increased antioxidant ability and decreased oxidative damage in the brain. Therefore, the pre-ischemic exercise alleviated brain damage and reduced motor dysfunction subsequent to MCAO. These results could be beneficial for the development of rational programs of prevention and treatment for ischemic stroke.

\section{Acknowledgements}

The present study was supported by a grant from the National Natural Science Foundation of China (no. 81201512).

\section{References}

1. Stummer W, Baethmann A, Murr R, et al: Cerebral protection against ischemia by locomotor activity in gerbils. Underlying mechanisms. Stroke 26: 1423-1430, 1995.

2. Ang ET, Wong PT, Moochhala S and Ng YK: Neuroprotection associated with running: is it a result of increased endogenous neurotrophic factors? Neuroscience 118: 335-345, 2003.

3. Endres M, Gertz K, Lindauer U, et al: Mechanisms of stroke protection by physical activity. Ann Neurol 54: 582-590, 2003.

4. Li J, Luan X, Clark JC, et al: Neuroprotection against transient cerebral ischemia by exercise pre-conditioning in rats. Neurol Res 26: 404-408, 2004.

5. Ding YH, Ding Y, Li J, et al: Exercise pre-conditioning strengthens brain microvascular integrity in a rat stroke model. Neurol Res 28: 184-189, 2006.

6. Zhang F, Wu Y and Jia J: Exercise preconditioning and brain ischemic tolerance. Neuroscience 17: 170-176, 2011.

7. Radak Z, Toldy A, Szabo Z, et al: The effects of training and detraining on memory, neurotrophins and oxidative stress markers in rat brain. Neurochem Int 49: 387-392, 2006.

8. Radák Z, Kaneko T, Tahara S, et al: Regular exercise improves cognitive function and decreases oxidative damage in rat brain. Neurochem Int 38: 17-23, 2001

9. Somani SM, Ravi R and Rybak LP: Effect of exercise training on antioxidant system in brain regions of rat. Pharmacol Biochem Behav 50: 635-639, 1995.

10. Somani SM and Husain K: Interaction of exercise training and chronic ethanol ingestion on antioxidant system of rat brain regions. J Appl Toxicol 17: 329-336, 1997.

11. Lappalainen Z, Lappalainen J, Oksala NK, et al: Diabetes impairs exercise training-associated thioredoxin response and glutathione status in rat brain. J Appl Physiol (1985) 106: 461-467, 2009.

12. Bromont $\mathrm{C}$, Marie $\mathrm{C}$ and Bralet $\mathrm{J}$ : Increased lipid peroxidation in vulnerable brain regions after transient forebrain ischemia in rats. Stroke 20: 918-924, 1989.

13. Kondo Y, Asanuma M, Nishibayashi S, et al: Late-onset lipid peroxidation and neuronal cell death following transient forebrain ischemia in rat brain. Brain Res 772: 37-44, 1997.

14. Re G, Azzimondi G, Lanzarini C, et al: Plasma lipoperoxidative markers in ischaemic stroke suggest brain embolism. Eur J Emerg Med 4: 5-9, 1997.

15. Hamakawa M, Ishida A, Tamakoshi K, et al: Repeated short-term daily exercise ameliorates oxidative cerebral damage and the resultant motor dysfunction after transient ischemia in rats. J Clin Biochem Nutr 53: 8-14, 2013.

16. Longa EZ, Weinstein PR, Carlson S and Cummins R: Reversible middle cerebral artery occlusion without craniectomy in rats. Stroke 20: 84-91, 1989.

17. Oyanagui Y: Reevaluation of assay methods and establishment of kit for superoxide dismutase activity. Anal Biochem 142: 290-296, 1984

18. Giannopolitis CN, Ries SK. Superoxide dismutases: I. Occurrence in higher plants. Plant Physiol 59: 309-314, 1977.

19. Ohkawa H, Ohishi $\mathrm{N}$ and Yagi K: Assay for lipid peroxides in animal tissues by thiobarbituric acid reaction. Anal Biochem 95: 351-358, 1979.

20. National Center for Health Statistics (US): Health, United States, 2010: With Special Feature on Death and Dying. Public Health Service. Report no. 2011-1232. US Department of Health and Human Services, Hyattsville, MD, 2011.

21. Krarup LH, Truelsen T, Gluud C, et al: Prestroke physical activity is associated with severity and long-term outcome from first-ever stroke. Neurology 71: 1313-1318, 2008. 
22. Deplanque D, Masse I, Lefebvre C, et al: Prior TIA, lipid-lowering drug use, and physical activity decrease ischemic stroke severity. Neurology 67: 1403-1410, 2006.

23. Sacco RL, Gan R, Boden-Albala B, et al: Leisure-time physical activity and ischemic stroke risk: the Northern Manhattan Stroke Study. Stroke 29: 380-387, 1998.

24. Stroud N, Mazwi TM, Case LD, et al; Ischemic Stroke Genetics Study Investigators: Prestroke physical activity and early functional status after stroke. J Neurol Neurosurg Psychiatry 80 1019-1022, 2009.

25. Rist PM, Lee IM, Kase CS, et al: Physical activity and functional outcomes from cerebral vascular events in men. Stroke 42: 3352-3356, 2011.

26. Guo M, Cox B, Mahale S, et al: Pre-ischemic exercise reduces matrix metalloproteinase-9 expression and ameliorates blood-brain barrier dysfunction in stroke. Neuroscience 151: 340-351, 2008.

27. Ding Y, Li J, Luan X, et al: Exercise pre-conditioning reduces brain damage in ischemic rats that may be associated with regional angiogenesis and cellular overexpression of neurotrophin. Neuroscience 124: 583-591, 2004.
28. Ding YH, Li J, Yao WX, et al: Exercise preconditioning upregulates cerebral integrins and enhances cerebrovascular integrity in ischemic rats. Acta Neuropathol 112: 74-84, 2006.

29. Zhang F, Jia J, Wu Y, et al: The effect of treadmill training pre-exercise on glutamate receptor expression in rats after cerebral ischemia. Int J Mol Sci 11: 2658-2669, 2010.

30. Zhang F, Wu Y, Jia J and Hu YS: Pre-ischemic treadmill training induces tolerance to brain ischemia: involvement of glutamate and ERK1/2. Molecules 15: 5246-5257, 2010.

31. Cheeseman KH and Slater TF: An introduction to free radical biochemistry. Br Med Bull 49: 481-493, 1993.

32. Esterbauer H: Estimation of peroxidative damage. A critical review. Pathol Biol (Paris) 44: 25-28, 1996.

33. Romero FJ, Bosch-Morell F, Romero MJ, et al: Lipid peroxidation products and antioxidants in human disease. Environ Health Perspect 106 (Suppl 5): 1229-1234, 1998.

34. Radak Z, Kumagai S, Taylor AW, et al: Effects of exercise on brain function: role of free radicals. Appl Physiol Nutr Metab 32: 942-946, 2007. 